\title{
SOLUTION MATCHING FOR BOUNDARY VALUE PROBLEMS FOR LINEAR EQUATIONS
}

\section{JOHNNY HENDERSON}

\author{
Departinent of Algebra, Combinatorics and Analysis \\ Auburn University \\ Auburn, Alabama 36849 U.S.A.
}

(Received March 17, 1988)

ABSTRACT. For the linear differential equation, $y^{(n)}=\sum_{1=1}^{n} a_{i}(x) y^{(1-1)},(1.1)$, where $n \geq 3$, solutions of multipoint boundary value problems on an interval [a,c] are obtained, via the use of Liapunov-1ike functions, by matching solutions of certain boundary value problems for (1.1) on $[a, b]$ with solutions of other boundary value problems for (1.1) on $[b, c]$.

KEY WORDS AND PHRASES. Multipolnt boundary value problem, solution matching, linear equation.

1980 MATHEMATICS SUBJECT CLASSIFICATION. 34B05, 34B10.

1. INTRODUCTION.

We will be concerned with the existence of solutions of $k$-point boundary value problems, $2<k \leq n$, on an interval $[a, c]$ for the $n$th order linear differential equation

$$
y^{(n)}=\sum_{i=1}^{n} a_{1}(x) y^{(1-1)}, n \geq 3
$$

where the $a_{1}(x) \in C[a, c]$.

The point $b \varepsilon(a, c)$ will fixed throughout, and we will employ techniques which match a solution $y_{1}(x)$ of a $(k-1)$-point boundary value problem for (1.1) on $[a, b]$ with a solution $y_{2}(x)$ of a 2-point boundary value problem for (1.1) on $[b, c]$ such that, $y(x)$ defined by

$$
y(x)=\left\{\begin{array}{l}
y_{1}(x), a \leq x \leq b, \\
y_{2}(x), b \leq x \leq c,
\end{array}\right.
$$

is a solution of a k-point boundary value problem for (1.1) on $[a, c]$. 
Solution matching techniques were first applied by Bailey, Shampine, and Waltman [1] where they dealt with solutions of 2-point boundary value problems for the second order equation $y^{\prime \prime}=f\left(x, y, y^{\prime}\right)$ by matching solutions of initial value problems. Since then, a number of papers have appeared in which solutions of 3-point boundary value problems on $[a, c]$ for the $n$th order ordinary differential equation $y^{(n)}=f\left(x, y, y^{\prime}, \ldots, y^{(n-1)}\right)$ were obtained by matching solutions of 2-point probleins on $[a, b]$ with 2-point problems on $[b, c]$; see, for example, Barr and Sherman [2], Das and Lall1 [3], Henderson [4], Moorti and Garner [5], and Rao, Murthy and Rao [6]. In those papers [2 - 6], a monotonicity condition imposed on $f$ plays an important role in the solution matching techniques in obtaining solutions of the 3-point problems. For example, in obtaining solutions of certain 3-point conjugate problems, Barr and Sherman [2] assumed that $f$ satisfies the conditions:

(1) $u_{n-1}<v_{n-1}$, and $(-1)^{n-j}\left(u_{j}-v_{j}\right) \geq 0, j=1,2, \ldots, n-2$, 1mp1y $f\left(x, u_{1}, \ldots, u_{n-1}, w\right)<f\left(x, v_{1}, \ldots, v_{n-1}, w\right)$, for all $x \in(a, b]$ and all w $\varepsilon R$, and

(11) $u_{n-1}<v_{n-1}$, and $u_{j} \leq v_{f}, j=1,2, \ldots, n-2$, imply $f\left(x, u_{1}, \ldots, u_{n-1}, w\right)<f\left(x, v_{1}, \ldots, v_{n-1}, w\right)$, for all $x \varepsilon[b, c)$ and all w $\varepsilon R$. Moorti and Garner [5], by assuming (i) and (1i) with respect to third order equations, obtained solutions of certain 3-point focal problems by matching solutions. Das and Lall1 [3] also assumed (1) and (11) with respect to third order equations, (however in relaxing other assumptions which were made in [2], the proof of Theorem 2.1 in [3] does not appear to be val1d). In the paper by Rao, Murthy and Rao [6], conditions (1) and (11) were modified some and solution matching was applied to obtain solutions of 3-point conjugate problems for third order ordinary differential equations. Then Henderson [4] generalized the monotonicity conditions of [6] and used solution matching to obtain the existence of solutions of a large class of 3 -polnt boundary value problems on $[a, c]$ for $y^{(n)}=f\left(x, y, y^{\prime}\right.$, ..., y $\left.{ }^{(n-1)}\right)$.

However, to obtain solutions of $k$-point problems on $\lfloor a, c\rfloor$, for $3<k \leq n$, by matching a solution $y_{1}(x)$ on $[a, b]$ with a solution $y_{2}(x)$ on $[b, c]$ under assumptions (1) and (11) or the more general monotonicity conditions in [4], one cannot necessarily conclude that $y_{1}(n-2)(b)=y_{2}^{(n-2)}(b)$, hence cannot conclude a solution on $[a, c]$. For the linear equation (1.1), using Liapunov-1ike functions, Barr and Miletta [7]matched solutions of (n-1)-point boundary value problems with solutions of 2-point boundary value problems, thus obtaining solutions of n-point boundary value problems. In this paper, using techniques similar to those of Barr and Miletta [7], we obtaln solutions of $k$-point boundary value problems for (1.1) on $[a, c]$. In particular, given $2<k \leq n$, positive integers $m_{1}, \ldots, m_{k}$ such that $\sum_{1=1}^{k} m_{1}=n$ and $m_{k}=1$, points $a \leq x_{1}<\ldots<x_{k-1}=b<x_{k} \leq c$, and $y_{1 j} \varepsilon R$, $0 \leq 1 \leq m_{j}-1,1 \leq j \leq k$, we are interested in solutions of boundary value problems for (1.1) satisfying 
$y^{(1)}\left(x_{j}\right)=y_{1 j}, 0 \leq 1 \leq m_{j}-1,1 \leq j \leq k-1, y\left(x_{k}\right)=y_{0, k}$, and for $\mu \varepsilon\{0,1, \ldots, n-2\}$ fixed,

$$
y^{(1)}\left(x_{j}\right)=y_{1 j}, 0 \leq 1 \leq m_{j}-1,1 \leq j \leq k-1, y^{(\mu)}\left(x_{k}\right)=y_{0, k} .
$$

The existence of unlque solutions of certaln (k-1)-point and 2-point boundary value problems for (1.1) used in the matching to obtain solutions of $(1.1),(1.2)$ and $(1.1),(1.3)$ will be established through the use of Liapunov-like or control functions.

DEFINITIONS. Given $M \geq 0$ and $[\alpha, \beta] \subseteq[a, c]$, a control function

$$
v_{11}\left(x, y_{1}, \ldots, y_{n}\right):[\alpha, \beta] \times R^{n}+R
$$

is a function which is continuous, locally Lipschitz with respect to $\left(y_{1}, \ldots, y_{n}\right)$ and satisfies

$$
\begin{aligned}
& \text { 1) } v_{M}\left(x, y_{1}, \ldots, y_{n}\right)=0 \text {, if } y_{n-1}=M \text {, } \\
& \text { 11) } v_{M}\left(x, y_{1}, \ldots, y_{n}\right)>0 \text {, if } y_{n-1}>M \text {. }
\end{aligned}
$$

Corresponding to $v_{i 1}\left(x, y_{1}, \ldots, y_{n}\right)$ and a solution $y(x)$ of the differential equation $(1.1)$, def Ine

$$
\begin{aligned}
V_{M}^{\prime}\left(x, y(x), \ldots, y^{(n-1)}(x)\right)=\operatorname{lin}_{h \rightarrow 0+} \operatorname{lnf}_{h} & \frac{1}{M}\left[V_{M}\left(x+h, y(x+h), \ldots, y^{(n-1)}(x+h)\right)\right. \\
& \left.-V_{M}\left(x, y(x), \ldots, y^{(n-1)}(x)\right)\right] .
\end{aligned}
$$

Extensive use will be made of the following lemma. Its proof is a simple extension of the one given for $n=1$ in Yoshizawa [3].

LEMMA 1.1. Suppose that $y(x)$ is a solution of (1.1), and that for some $M \geq 0$ and $[\alpha, \beta] \subseteq[a, c], v_{M}\left(x, y_{1}, \ldots, y_{n}\right)$ is a control function. Then $v_{M}\left(x, y(x), \ldots, y^{(n-1)}(x)\right)$ is nondecreasing, (nonincreasing), if and only if $V_{M}^{\prime}\left(x, y(x), \ldots, y^{(n-1)}(x)\right) \geq 0, \quad\left(V_{M}^{\prime}\left(x, y(x), \ldots, y^{(n-1)}(x)\right) \leq 0\right)$.

In section 2, we carry out the construction for matching solutions of $(k-1)$-point boundary value problems with 2-point boundary value problems in obtainIng a solution of $(1.1),(1.2)$. Then in section 3 , results completely analogous to those obtained in section 2 are stated for solutions of (1.1), (1.3).

2. EXISTENCE OF SOLUTIONS OF (1.1), (1.2).

In this section, for $2<k \leq n$, let $m_{1}, \ldots, m_{k}$ be positive integers such that

$\sum_{i=1}^{k} m_{1}=n$ and $m_{k}=1$. Let $a \leq x_{1}<\ldots<x_{k-1}=b<x_{k} \leq c$ and $y_{1 j} \varepsilon R$, 
$0 \leq 1 \leq m_{j}-1,1 \leq j \leq k$ be given. We match solutions $y(x)$ of (1.1) satisfying

$$
y^{(1)}\left(x_{j}\right)=y_{1 j}, 0 \leq 1 \leq m_{j}-1,1 \leq j \leq k-1, y^{(n-1)}\left(x_{k-1}\right)=m,
$$

with solutions $z(x)$ of $(1.1)$ satisfying

$$
z^{(1)}\left(x_{k-1}\right)=y^{(1)}\left(x_{k-1}\right), 0 \leq 1 \leq n-3, z^{(n-1)}\left(x_{k-1}\right)=m, z\left(x_{k}\right)=y_{0, k}
$$

where $n \in R$, to obtain a solution of (1.1), (1.2). The use made of a fanily of control functions in establishing existence of unlque solutions of $(k-1)$-point and 2-point problems and in establishing desirel monotone properties is seen in the next four theorems.

THEOREM 2.1. Assuine that there exists a control function $v_{0}\left(x, y_{1} \ldots, y_{n}\right)$ on $[a, b]$ such that $V_{0}^{\prime}\left(x, y(x), \ldots, y^{(n-1)}(x)\right) \geq 0$, for all solutions $y(x)$ of $(1.1)$. Then for each $m \varepsilon R$, the boundary value problen for (1.1) satisfying

$$
y^{(1)}\left(x_{j}\right)=y_{1 j}, 0 \leq 1 \leq m_{j}-1,1 \leq j \leq k-1, y^{(n-2)}\left(x_{k-1}\right)=m,
$$

has a unique solution.

PROOF. It suffices to show that the boundary value problen for (1.1) satisfyIng $y^{(1)}\left(x_{j}\right)=0,0 \leq 1 \leq m_{j-1}, 1 \leq j \leq k-1, y^{(n-2)}\left(x_{k-1}\right)=0$, has only the trivial solution. Assume on the contrary that this boundary value problen has a nontrivial solution $y(x)$. It follows that there exist points $x_{1}<\tau_{1}<\tau_{2}<\tau_{3} \leq x_{k}$ such that $y^{(n-2)}\left(\tau_{1}\right)=y^{(n-2)}\left(\tau_{3}\right)=y^{(n-1)}\left(\tau_{2}\right)=0$, and $y^{(n-2)}(x)$ or $-y^{(n-2)}(x)$ has a positive local maximum at $x=\tau_{2}$.

Assume without loss of generality that $y^{(n-2)}(x)$ has a positive local maximum at $x=\tau_{2}$. Now from our hypotheses, $v_{0}\left(\tau_{1}, y\left(\tau_{1}\right), \ldots, y^{(n-1)}\left(\tau_{1}\right)\right)$ $=v_{0}\left(\tau_{3}, y\left(\tau_{3}\right), \ldots, y^{(n-1)}\left(\tau_{3}\right)\right)=0$ and $v_{0}\left(\tau_{2}, y\left(\tau_{2}\right), \ldots, y^{(n-1)}\left(\tau_{2}\right)\right)>0$. However, since $v_{0}^{\prime}\left(x, w(x), \ldots, y^{(n-1)}(x)\right) \geq 0, v_{0}\left(x, y(x), \ldots, y^{(n-1)}(x)\right)$ is nondecreasing; consequently, $v_{0}\left(\tau_{3}, y\left(\tau_{3}\right), \ldots, y^{(n-1)}\left(\tau_{3}\right)\right)>0$ which is a contradiction. Thus, the assertion of the theoren is true.

THEOREM 2.2. Assume that for each $m \in R$, there exists a solution $y_{1}(x, m)$ of boundary value problem (1.1), (2.1). If for each $M \geq 0$, there exists a control function $v_{M}\left(x, y_{1}, \ldots, y_{n^{\prime}}\right)$ on $[a, b]$ such that $v_{M}^{\prime}\left(x, y(x), \ldots, y^{(n-1)}(x)\right) \geq 0$ for all solutions $y(x)$ of $(1.1)$, then $y_{1}{ }^{(n-2)}\left(x_{k-1}, m\right)$ is a strictly increasing function of $\mathrm{m}$.

PROOF. Let $m_{1}<m_{2}$ and assume $y_{1}^{(n-2)}\left(x_{k-1}, m_{2}\right) \leq y_{1}^{(n-2)}\left(x_{k-1}, m_{1}\right)$. Then consider the nontrivial solution $w(x) \equiv y_{1}\left(x, m_{1}\right)-y_{1}\left(x, m_{2}\right)$ of $(1.1)$. It follows that $w^{(n-2)}\left(x_{k-1}\right) \geq 0$.

Since $w^{(1)}\left(x_{j}\right)=0,0 \leq 1 \leq m_{j}-1,1 \leq j \leq k-1$, it follows by successive 


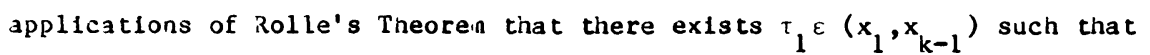
${ }_{N}^{(n-2)}\left(\tau_{1}\right)=0$. Yet, from $w^{(n-2)}\left(\tau_{1}\right)=0$, the assumption that $w^{(n-2)}\left(x_{k-1}\right) \geq 0$, and the fact that by construction $w^{(n-1)}\left(x_{k-1}\right)=m_{1}-m_{2}<0$, we have that there exists ${ }^{\prime} 2^{\varepsilon}\left(\tau_{1}, x_{k-1}\right)$ such that ${ }^{(n-1)}\left(\tau_{2}\right)=0$ and ${ }^{(n-2)}(x)$ has a positive local inaximum at $x=\tau_{2}$. Now, let $\tau_{1}<\alpha<\tau_{2}<\beta<x_{k-1}$ be such that $w^{(n-2)}(\alpha)=w^{(n-2)}(\beta)$ $=M$ and $w^{(n-2)}\left(\tau_{2}\right)>M$. Since there exists a control function $V_{M}$, we have $v_{M}\left(\alpha, w(\alpha), \ldots, w^{(n-1)}(\alpha)\right)=v_{M}\left(\beta, w(\beta), \ldots, w^{(n-1)}(\beta)\right)=0$ and $v_{M}\left(\tau_{2}, w\left(\tau_{2}\right), \ldots, w^{(n-1)}\left(\tau_{2}\right)\right)>0$. However, since $V\left(x, w(x), \ldots, w^{(n-1)}(x)\right)$ is nondecreasing on $[a, b]$, it follows tnat $v_{M}\left(\beta, w(\beta), \ldots, w^{(n-1)}(\beta)\right)>v$; again a contradiction.

Thus, w ${ }^{(n-2)}\left(x_{k-1}\right)<0$, winich in turn implies that $y_{1}(n-2)\left(x_{k-1}, m_{1}\right)$ $<y_{1}(n-2)\left(x_{k-1}, m_{2}\right)$. The proof is coinplete.

REMARK. We remarik that, under the hypotheses of Theorem 2.2, it can be argued by finite induction that $y_{1}^{(n-j)}\left(x_{k-1}, m\right), 2 \leq j \leq n-m_{k-1}$, are all strictly increasing functions of $\mathrm{m}$.

The next two theorems follow from arguments very similar to those used in Theorems 2.1 and 2.2 .

THEOREM 2.3. Let $r_{m_{k-1}}, \ldots, r_{n-3}$ be given. Assume that there exists a control function $w_{0}\left(x, y_{1}, \ldots, y_{n}\right)$ on $[b, c]$ such that $w_{0}^{\prime}\left(x, y(x), \ldots, y^{(n-1)}(x)\right) \geq 0$ for all solutions $y(x)$ of (1.1). Then, for each $m \varepsilon R$, the boundary value problem for (1.1) satisfying

$$
y^{(1)}\left(x_{k-1}\right)=\left\{\begin{array}{ll}
y_{1, k-1}, & 0 \leq 1 \leq m_{k-1} \\
r_{1}, & m_{k-1} \leq 1 \leq n-3
\end{array}\right\}, y^{(n-2}\left(x_{k-1}\right)=m, y\left(x_{k}\right)=y_{0, k} \text {, }
$$

has a unique solution.

THEOREM 2.4. Let $y_{1}(x, m)$ be as in Theorem 2.2 and assuine that for each $m \varepsilon R$, there exists a solution $y_{2}(x, m)$ of boundary value problem $(1.1),(2.2)$. If for each $M \geq 0$, there exists a control function $w_{M}\left(x, y_{1}, \ldots y_{n}\right)$ on $[b, c]$ such that $W_{M}^{\prime}\left(x, y(x), \ldots, y^{(n-1)}(x)\right) \geq 0$ for all solutions $y(x)$ of $(1.1)$, then $y_{2}(n-2)\left(x_{k-1}, m\right)$ is a strictly decreasing function of $m$.

PROOF. Let $m_{1}<m_{2}$ and then set $w(x) \equiv y_{2}\left(x, m_{2}\right)-y_{2}\left(x, m_{1}\right)$. Then argue as in Theorein 2.2 that $w^{(n-2)}\left(x_{k-1}\right)<0$.

We are now prepared to match solutions and obtain a solution of $(1.1),(1.2)$. 
THEOREM 2.5. Assume that, for each in $\varepsilon R$, there exists a unique solution of $(1.1),(2.1)$ on $[a, b]$, and that, for each in $\varepsilon R$, there exists a solution of $(1.1),(2.2)$ on $[b, c]$. Assume, moreover, that the boundary value problem for (1.1) on $[b, c]$ satisfying

$$
y^{(1)}\left(x_{k-1}\right)=0,1=0, \ldots, n-3, n-1, y\left(x_{k}\right)=0,
$$

has only the trivial solution. If for each $M \geq 0$, there exist control functions $v(x, y, \ldots, y)$ and $w(x, y, \ldots, y)$ on $[a, b]$ and $[b, c]$ respectively, such that $V_{M}\left(x, y(x), \ldots, y^{(n-1)}(x)\right) \geq 0$ and $W_{M}^{\prime}\left(x, y(x), \ldots, y^{(n-1)}(x)\right) \geq 0$ for all solutions $y(x)$ of $(1.1)$, then the k-point boundary value problem (1.1), (1.2) has a solution on $[a, c]$.

PROOF. If $y_{1}(x, m)$ is a solution of the boundary value prodlen $(1.1),(2.1)$, then by Theorem 2.2, $y_{1}^{(n-2)}\left(x_{k-1}, m\right)$ is a strictly increasing function of $m$. We contend, furtherinore, that $y_{1}(n-2)\left(x_{k-1}, m\right)$ is a continuous function of m with range all of $R$. To see this, it suffices to show the latter; that is, $\left\{y_{1}^{(n-2)}\left(x_{k-1}, m\right) \mid m \varepsilon R\right\}=R$.

Thus, let $r \varepsilon R$. From Theoren 2.1, there is a unique solution $u(x)$ of $(1.1),(2.3)$ satisfying

$$
\begin{aligned}
& u^{(1)}\left(x_{j}\right)=y_{1 f}, 0 \leq 1 \leq m_{f}-1,1 \leq j \leq k-1, \\
& u^{(n-2)}\left(x_{k-1}\right)=r .
\end{aligned}
$$

Consider now the solution $w(x) \equiv u(x)-y_{1}\left(x, u^{(n-1)}\left(x_{k-1}\right)\right)$ of equation $(1,1)$. $w(x)$ satisfies the boundary conditions of type $(2.1)$,

and

$$
\begin{aligned}
& w^{(1)}\left(x_{j}\right)=0,0 \leq 1 \leq m_{j}-1,1 \leq j \leq k-1, \\
& w^{(n-1)}\left(x_{k-1}\right)=u^{(n-1)}\left(x_{k-1}\right)-u^{(n-1)}\left(x_{k-1}\right)=0 .
\end{aligned}
$$

By the hypotheses of the theorem, $w(x) \equiv 0$, and hence $u(x)=y_{1}\left(x, u^{(n-1)}\left(x_{k-1}\right)\right)$. Consequently, $y_{1}^{(n-2)}\left(x_{k-1}, u^{(n-1)}\left(x_{k-1}\right)\right)$ $=u^{(n-2)}\left(x_{k-1}\right)=r$, and it follows that $r \in\left\{y_{1}^{(n-2)}\left(x_{k-1}, m\right) \mid m \varepsilon R\right\}$. In summation, $y_{1}^{(n-2)}\left(x_{k-1}, m\right)$ is a strictly increasing, continuous function of $m$ with range all of $R$.

Similarly, if as in Theorem $2.4, y_{2}(x, m)$ is a solution of the boundary value problem (1.1), (2.2), then it will follow that, from the existence of unique solutions of $(1.1),(2.4)$ and $(1.1),(2.5), y_{2}(n-2)\left(x_{k-1}, m\right)$ is a strictly decreasing, continuous function of $m$ with range all of $R$. Thus, there is a unique $m_{0} \varepsilon R$, such that $y_{1}^{(n-2)}\left(x_{k-1}, m_{0}\right)=y_{2}^{(n-2)}\left(x_{k-1}, m_{0}\right)$. Then 


$$
y(x)= \begin{cases}y_{1}\left(x, t_{0}\right) & , a \leq x \leq b, \\ y_{2}\left(x, m_{0}\right) & , b \leq x \leq c,\end{cases}
$$

is a solution of the boundary value problem $(1,1),(1.2)$ on $[a, c]$.

3. EXISTENCE OF SOLUTIONS OF (1.1), (1.3).

In this section, let $k, m_{1}, \ldots, m_{k}, a \leq x_{1} \ldots<x_{k-1}=b<x_{k} \leq c$ and $y_{1 j} \varepsilon R$ be as in the previous section. Let $\mu \varepsilon\{0,1, \ldots, n-2\}$ be given. We state theorems analogous to those in section 2 in which solutions $y(x)$ of (1.1) satisfying

$$
y^{(1)}\left(x_{j}\right)=y_{1 j}, 0 \leq 1 \leq m_{j}-1,1 \leq j \leq k-1, y^{(n-1)}\left(x_{k-1}\right)=n,
$$

are matched with solutions $v(x)$ of $(1.1)$ satisfying

$$
v^{(1)}\left(x_{k-1}\right)=y^{(1)}\left(x_{k-1}\right), 0 \leq 1 \leq n-3, v^{(n-1)}\left(x_{k-1}\right)=1 n, v^{(\mu)}\left(x_{k}\right)=y_{0, k} \text {, }
$$

where $m \varepsilon R$, ylelding a solution of (1.1), (1.3). We will onit the proofs of these theoreins. Moreover, Theorens 2.1 and 2.2 are applicable in this section.

THEOREM 3.1. Assume the hypotheses of Theorem 2.3. Then, for each in $\varepsilon$, the boundary value problem for (1.1) satisfying

$y^{(1)}\left(x_{k-1}\right)=\left\{\begin{array}{ll}y_{1, k-1} & , 0 \leq 1 \leq m_{k-1}^{-1} \\ r_{1} & , m_{k-1} \leq 1 \leq n-3\end{array}\right\}, y^{(n-2)}\left(x_{k-1}\right)=m_{, y}^{(\mu)}\left(x_{k}\right)=y_{0, k}$,

has a unique solution

Theorem 3.2. Let $y_{1}(x, m)$ be as in Theorem 2.2 and assume that for each $m \varepsilon R$, there exists a solution $v(x, m)$ of boundary value problem (1.1), (3.1). If for each $M \geq 0$, there exists a control function $w_{M}\left(x, y_{1}, \ldots, y_{n}\right)$ on $[b, c]$ such that $W_{M}^{\prime}\left(x, y(x), \ldots, y^{(n-1)}(x)\right) \geq 0$ for all solutions $y(x)$ of $(1,1)$, then $v^{(n-2)}\left(x_{k-2}, m\right)$ is a strictly decreasing function of $\mathrm{m}$.

THEOREM 3.3. Assume that, for each $m \varepsilon R$, there exists a unique solution of (1.1), (2.1) on $[a, b]$, and that, for each $m \varepsilon R$, there exists a solution of $(1.1),(3.1)$ on $[b, c]$. Assume, moreover, that the boundary value problem for (1.1) on $[b, c]$ satisfying

$$
y^{(1)}\left(x_{k-1}\right)=0,1=0, \ldots, n-3, n-1, y^{(\mu)}\left(x_{k}\right)=0,
$$

has only the trivial solution. If for each $M \geq 0$, there exist control functions $v_{M}\left(x, y_{1}, \ldots, y_{n}\right)$ on $[a, b]$ and $w_{M}\left(x, y_{1}, \ldots, y_{n}\right)$ on $[b, c]$, such that $V_{M}^{\prime}\left(x, y(x), \ldots, y^{(n-1)}(x)\right) \geq 0$ and $W_{M}^{\prime}\left(x, y(x), \ldots, y^{(n-1)}(x)\right) \geq 0$ for all solutions $y(x)$ of (1.1), then the boundary value problem (1.1), (1.3) has a solution on [a,c]. 


\section{REFERENCES}

1. BAILEY, P., SHAMPINE, L. and WALTMAN, P. Nonlinear Two Point Boundary Value Problems, Acadentc Press, New York, 1968.

2. BARR, D. and SHERMAN T. Existence and uniqueness of solutions of three-point boundary value problems, J. Differential Equations 13 (1973), 197-212.

3. DAS, K. M. and LALLI, B. S. Boundary value problems for $y^{\prime \prime \prime}=f\left(x, y, y^{\prime} y^{\prime \prime}\right)$, J. Math. Anal. Appl. 81 (1981), 300-307.

4. HENDERSON, J. Three-point boundary value problems for ordinary differential equations by matching solutions, Nonlinear Anal. 구 (1933), 411-417.

5. MOORTI, V. R. G. and GARNER, J. B. Existence-uniqueness theorens for three-point boundary value problems for nth-order nonlinear differential equations, J. Differential Equations 29 (1978), 205-213.

6. RAO, D. R. K. S., MURTHY, K. N., and RAU, A. S. On three-point boundary value problems associated with third order differential equations, Nonlinear Anal. $\underline{5}$ $(1981), 669-673$.

7. BARR, D. and MILETTA, P. An existence and uniqueness criterion for solutions of boundary value problens, J. Differential Equations 16 (1974).

8. YOSHIZAWA, T. Stability Theory by Llapunov's Second Method, Publ. Math. Soc. Japan, No. 9, Math. Soc. Japan, Tokyo, 1966. 


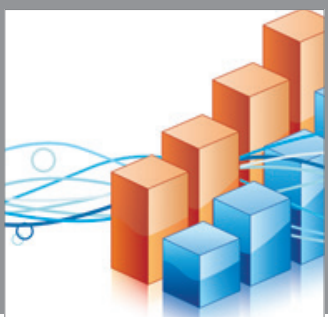

Advances in

Operations Research

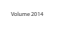

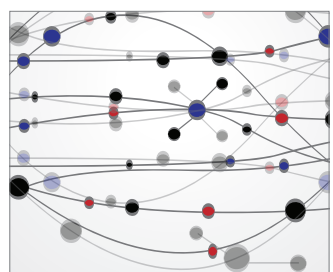

\section{The Scientific} World Journal
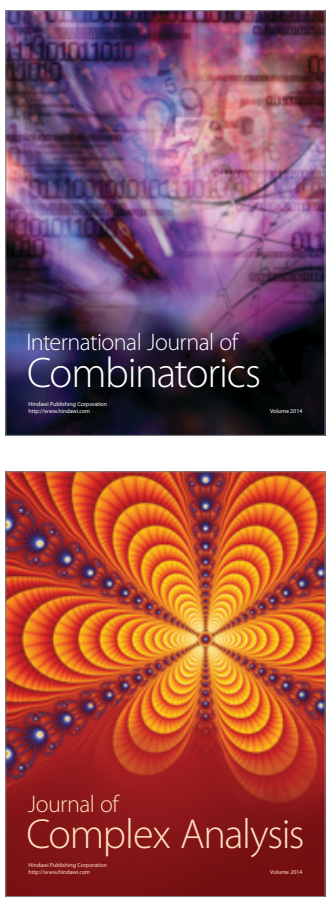

International Journal of

Mathematics and

Mathematical

Sciences
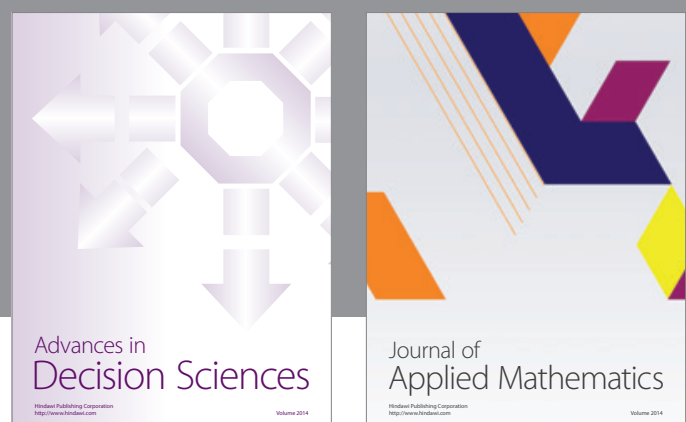

Journal of

Applied Mathematics
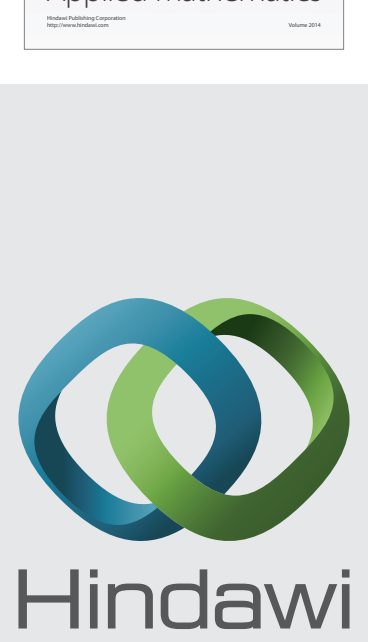

Submit your manuscripts at http://www.hindawi.com
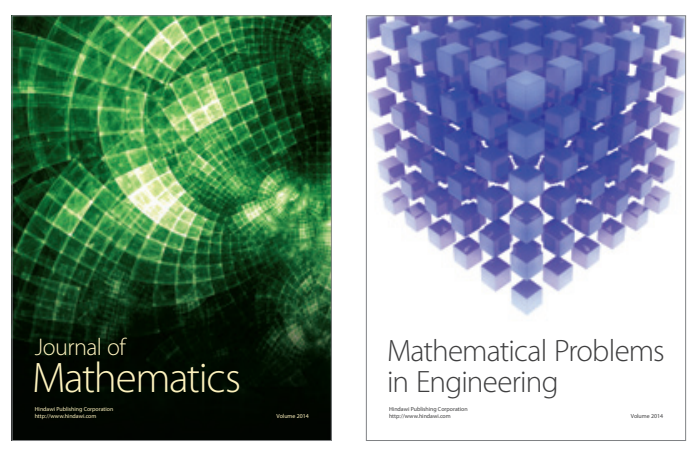

Mathematical Problems in Engineering
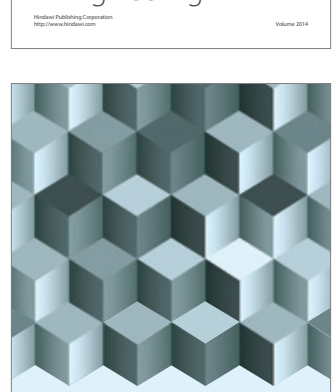

Journal of

Function Spaces
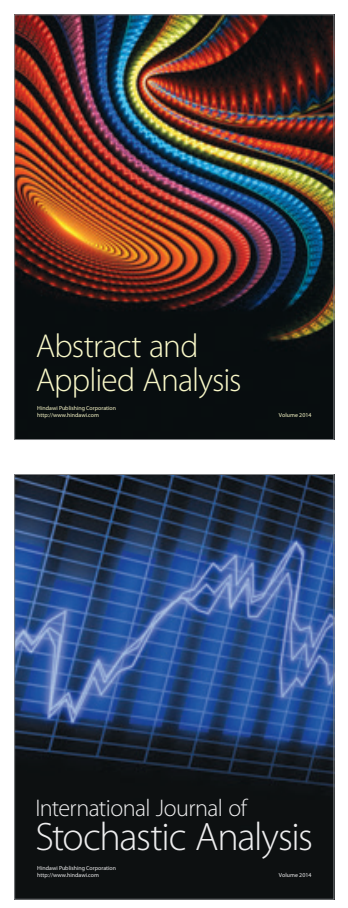

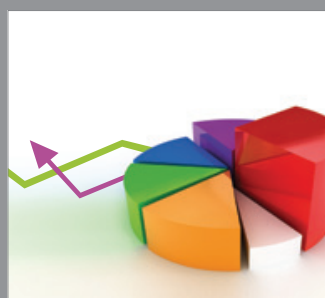

ournal of

Probability and Statistics

Promensencen
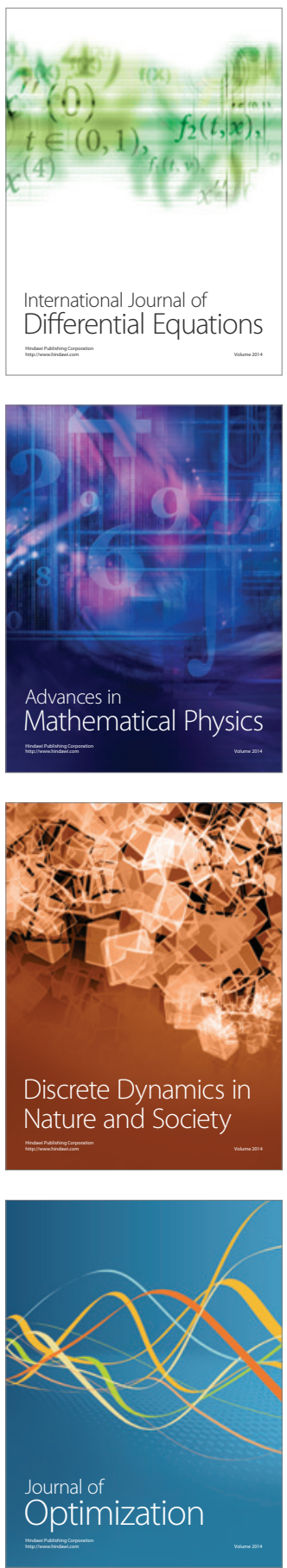